\title{
THE FIBER OPTIC PROBE HYDROPHONE
}

\author{
C. Wurster ${ }^{1}$, J. Staudenraus ${ }^{2}$, W. Eisenmenger ${ }^{1}$ \\ ${ }^{1}$ 1. Physikalisches Institut, Universität Stuttgart, Pfaffenwaldring 57, D-70550 Stuttgart, Germany \\ ${ }^{2}$ Richard Wolf GmbH, D-75438 Knittlingen, Germany
}

\begin{abstract}
We present a new type of hydrophone based on a fiber-optic sensor principle for shock wave and ultrasonic measurements in water. Its detection mechanism is based on the change of the optical reflection coefficient at the end surface of a glass fiber in water by pressure signals. The relation between the intensity of the reflected light and the water pressure is defined by material constants so that calibration by reference is not necessary. Shock wave measurements were made with the fiber optic probe hydrophone and compared with the results obtained by PVDF membrane and needle hydrophones. Important advantages are high resolution in space and time, a reproducible response also to negative pressure signals with high accuracy, high cavitation threshold, large bandwidth $(>1 \mathrm{GHz})$, ideal electromagnetic shielding, high deterioration resistance and simple calibration control.
\end{abstract}

\section{INTRODUCTION}

In recent years the demand for precise measurements of high positive and negative pressures in liquids has been increasing especially in medical science and biology. So far piezoelectric needle and membrane hydrophones with sensitive elements consisting of polarized polyvinyliden fluoride (PVDF) are widely used for all kinds of shock wave measurements. Examples for such applications are sound field scanning experiments of lithotripters [1] and diagnostic ultrasound instruments [2]. In this respect the new fiber optic probe hydrophone overcomes several limitations of conventional piezoelectric hydrophones. In addition to a more difficult calibration procedure PVDF hydrophones have a much worse perfor- mance relating to unprecise reproduction of high negative pressures, cavitation tendency, low bandwidth, bad electromagnetic shielding and ageing problems.

\section{DETECTION MECHANISM}

The acoustically sensitive element is the endface of a multimode glass fiber where a temporal pressure change in an acoustic wavefield is detected by a change of the optical reflection coefficient. A rise of the acoustic pressure will lead to a compression of the surrounding liquid resulting in an increase of the refraction index. The change of the refraction index of quartz glass can be neglected because of its low compressibility. With these assumptions the coefficient of reflection $R$ is calculated from the refractive indices of the liquid $n$ and the fiber core $n_{c}$ using the Fresnel equations. A good approximation can be achieved if light in cidence perpendicular to the fiber endface is considered:

$$
R=\left[\left(n_{c}-n\right)^{2} /\left(n_{c}+n\right)^{2}\right]
$$

This principle of pressure detection gives rise to a theoretical bandwidth which is only limited by the decay length of the evanescent mode [3] of the reflected part of the incident light wave at the glass-liquid boundary. With a typical decay length of about half the optical wavelength, bandwidths larger than $1 \mathrm{GHz}$ are possible. Due to limitations of the electronic equipment bandwidths in the $\mathrm{GHz}$-range have not yet been achieved.

\section{CALIBRATION}

Within the scope of shock wave experiments in water the relation between pressure and reflection coefficient is strictly defined by material con- 
stants listed in various publications $[4,5,6]$. In this case calibration by reference is not necessary. The relation between refraction index $n_{w}$ and water pressure $p$ is defined by the isentropic Tait equation connecting water pressure and mass density $[5,6]$ and the Gladstone-Dale relation connecting mass density and refraction index. Combining both relations and using the Tait parameters $(Q=295.5 \mathrm{MPa}, \gamma=7.44)$ under static conditions $\left(p_{0}=0.1 \mathrm{MPa}, n_{w, 0}=1.329\right)$ the following equation between the refraction index of water $n_{w}$ and the acoustic pressure $p$ is obtained:

$$
n_{w}(p)=1+\left(n_{w, 0}-1\right)\left(1+\frac{p-p_{0}}{p_{0}+Q}\right)^{1 / \gamma}
$$

The relation between the change of reflectivity $\Delta R$ and pressure $p$ can be derived from equations (1),(2) conserving all non-linearities:

$$
\Delta R(p)=\frac{\left(n_{c}-n_{w}(p)\right)^{2}}{\left(n_{c}+n_{w}(p)\right)^{2}}-\overbrace{\frac{\left(n_{c}-n_{w, 0}\right)^{2}}{\left(n_{c}+n_{w, 0}\right)^{2}}}^{R_{0}}
$$

The change in reflectivity in relation to the static reflectivity $\Delta R / R_{0}$ is measured as a change of a photodiode signal in relation to the static photodiode signal $\Delta U / U_{0}$. Due to the non-ideal experimental set-up it is necessary to take into account an additional static reflectivity $\alpha \cdot R_{0}$ caused by internal reflections and coupler crosstalk in the fiber optic system. With this assumption the dependence of the photodiode signal on the pressure and the sensitivity $H$ of the fiber optic probe hydrophone can be defined $\left(\Delta p=p-p_{0}\right)$ :

$\frac{\Delta U}{U_{0}}=\frac{\Delta R}{(1+\alpha) R_{0}}=\frac{\Delta R \cdot \Delta p}{(1+\alpha) R_{0} \cdot \Delta p}=H \cdot \Delta p$

Figure 1 shows the dependence of $H$ on the acoustic pressure for an ideal experimental setup with no internal reflections $(\alpha=0)$. The nonlinearity of the system is below $5 \%$ within the pressure values $(-12 \mathrm{MPa}-40 \mathrm{MPa})$ appearing in common shock wave experiments. Thus it is reasonable to use an average value of the sensitivity $H_{0}=-1.95 \cdot 10^{-3} \mathrm{MPa}^{-1}$ neglecting the weak pressure dependence. The reduction of $H$ due to

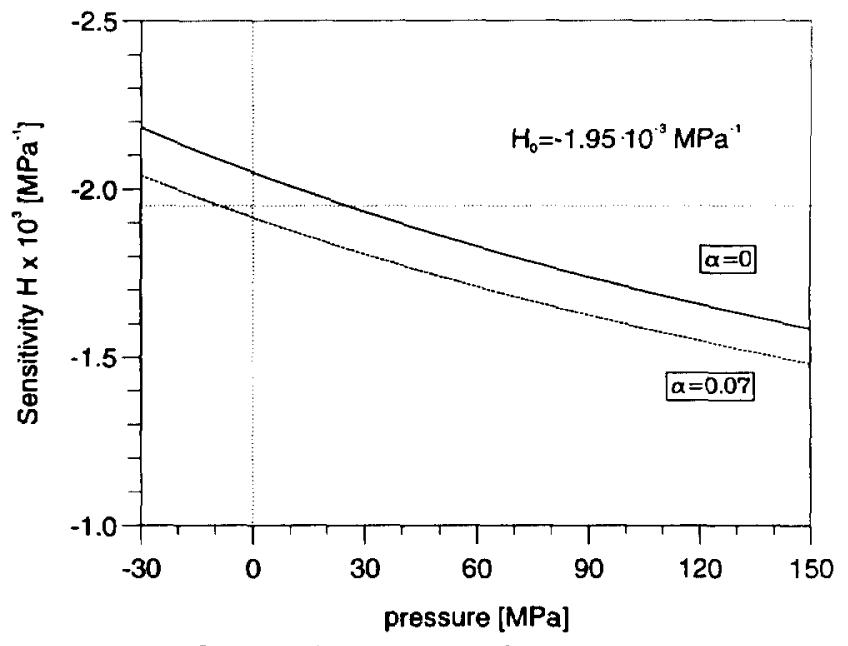

Figure 1: Sensitivity of the fiber optic probe hydrophone versus water pressure in an ideal environment $(\alpha=0)$ and with an additional static reflectivity $\alpha=0.07 \cdot R_{0}$ caused by non-idealities in the fiber optic system.

non-idealities in the fiber optic system is illustrated in Figure 1 for an additional static reflectivity $\alpha \cdot R_{0}=0.07 \cdot R_{0}$. The zero-reflectivity $\alpha \cdot R_{0}$ is measured by dipping the fiber end into an immersion liquid of the same refraction index as the glass fiber. Typical values for $\alpha$ vary from $0.03-0.1$.

Great importance has to be attached to a clean and smooth fiber endface otherwise $\Delta R$ and $R_{0}$ are reduced by surface scattering. In this case a drastic decrease of the signal to noise ratio is observed although $\Delta R / R_{0}$ and the sensitivity $H$ remain unchanged. The best fiber endfaces have been prepared by cutting the fiber with a commercial fiber separator whereas polishing of the fiber endface produced much worse results.

\section{TECHNICAL DESCRIPTION}

The fiber optic probe hydrophone is mounted in a compact and transportable unit. A sketch of its experimental set-up is shown in Figure 2. A fiberpigtailed $100 \mathrm{~mW}$-GaAlAs-laser diode is used as light source (wavelength: $812 \mathrm{~nm}$ ). The laser pigtail consists of $100 / 140 \mu \mathrm{m}$ step index multimode glass fiber which is guided through a commercial $3 \mathrm{~dB}$ X-fiber-coupler. The free end of the fiber is introduced into the water tank. The fiber is guided through a cannula where it is fixed with clamps. The cannula itself is tightly fixed to an external 


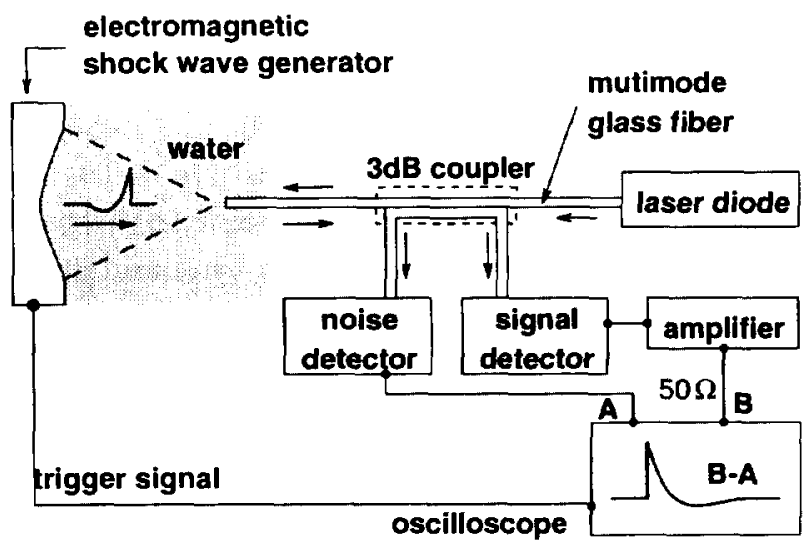

Figure 2: Sketch of the experimental set-up of the fiber optic probe hydrophone.

tripod in order to avoid distortion of the whole set-up under the influence of shock waves.

Half of the reflected light intensity is decoupled and guided to the signal photodetector consisting of a $\mathrm{p}-\mathrm{i}$-n-diode connected with an amplifier (bandwidth $20 \mathrm{MHz}$ ) also used as impedance converter from the $\mathrm{k} \Omega$ range to $50 \Omega$. The output signal is recorded with a sampling oscilloscope including an option on digital data acquisition.

In order to reduce the influence of laser noise on the signal half of the primary coherent laser intensity is decoupled and guided to the noise detector. The noise signal is scaled to the noise level of the reflected signal and subtracted.

\section{SHOCK WAVE EXPERIMENTS}

The response of a fiber optic probe hydrophone to an incident shock wave accompanied by a huge negative pressure phase is illustrated in Figure 3. The measured rise time of the shock front is determined by the limited bandwidth of the electronic equipment whereas the physical bandwidth lies in the nanosecond range [7].

The adjacent overshoot structure is caused by the interference of the incoming shock wave, the acoustically rigid reflected wave and the wave diffracted at the hydrophone edge doubling the water pressure for some tens of nanoseconds [8]. As shown in Figure 3 this overshoot can easily be eliminated by linear extrapolation of the pressure decay signal. The adjacent negative pressure

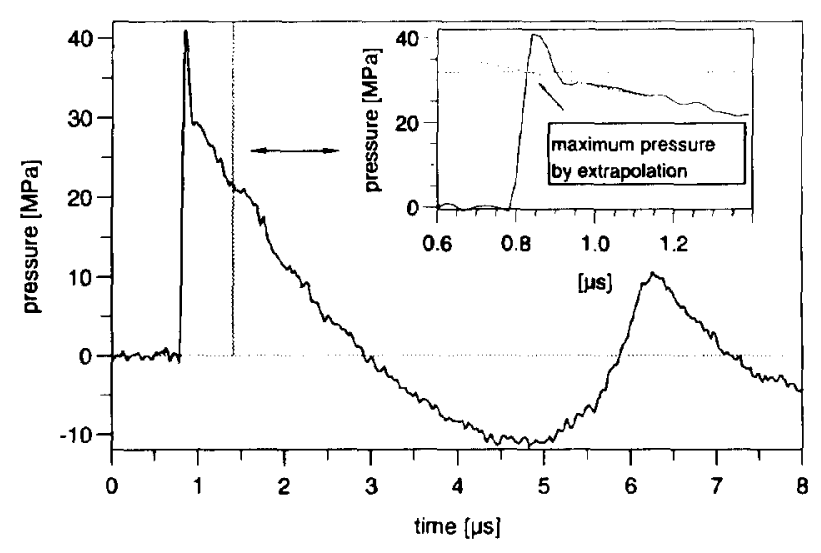

Figure 3: Typical shock wave signal obtained by the fiber optic probe hydrophone. The overshoot at the end of the signal increase is caused by interference with the acoustically rigid reflected wave [8]. Maximum pressure can be determined by extrapolation. phase is reproduced down to pressures of $-12 \mathrm{MPa}$.

The advantages of the fiber optic hydrophone can be demonstrated by comparing with the results of PVDF membrane and needle hydrophones under identical conditions. In Figure 4 a the overshoot at the shock front is already eliminated. The negative pressure phase can reproducibly be displayed only with the fiber optic probe hydrophone (Figure 4a) whereas PVDF membrane and needle hydrophones display only the leading edge of the negative pressure phase. A low cavitation thresh-

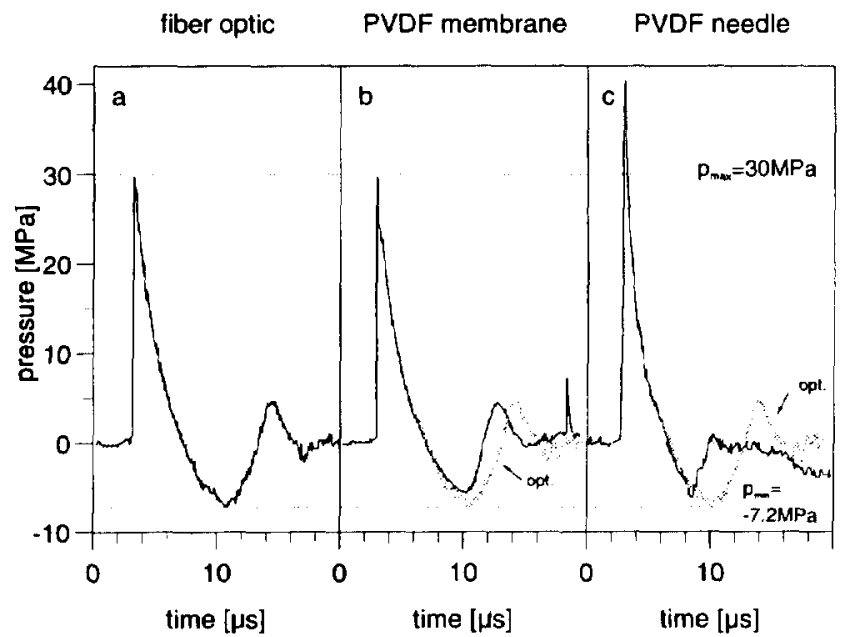

Figure 4: Comparison of shock wave measurements under identical parameters done with the fiber optic, the PVDF-membrane and a commercial PVDF needle hydrophone. 
old promotes a detachment of the water film resulting in a too low and non-reproducible display of negative pressure signals (Figure $4(\mathrm{~b}, \mathrm{c})$ ) using the optical hydrophone as reference (grey curve).

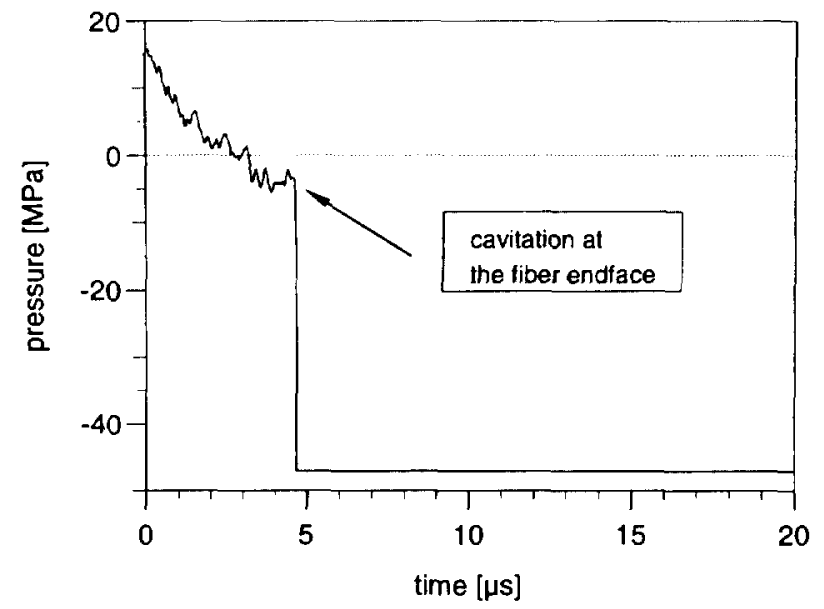

Figure 5: Cavitation at the fiber endface of the fiber optic probe hydrophone. The photodiode reaches the saturation limit because of reflection at a glass/water vapor boundary.

The high adhesion of water to glass is responsible for the high cavitation threshold of the optical probe hydrophone. Nevertheless if cavitation occurs at the fiber endface the reflection coefficient will change dramatically because reflection takes place at a glass/water vapor boundary. Figure 5 shows the corresponding abrupt change of the photodiode signal to the saturation limit. This behaviour makes it possible to identify cavitation safely in case it takes place at the fiber tip.

\section{FURTHER ADVANTAGEOUS PROPERTIES}

The fiber optic probe hydrophone guarantees ideal electromagnetic shielding based on its optical detection mechanism which allows the installation of the electronic instrumentation far away from the detection area in the water tank.

Fiber damage and a dirty or rough fiber endface can be found by monitoring the static reflectivity. Repair efforts are mostly confined to prepare a new endface by cleaving the fiber again, a new calibration is not necessary.

The low cavitation threshold and high resistance to distortion provide a considerable longterm stability. No ageing with respect to reliability and calibration have been observed.

\section{CONCLUSION}

The fiber optic probe hydrophone is a a well-suited tool for sound measurements in liquids especially in an environment where high positive or negative pressures are expected. It is superior to piezoelectric hydrophones with respect to time and spatial resolution and its reliability in measuring negative pressures. Additionally cavitation on the sensitive area can be identified safely by a drastic change of reflectivity. Calibration by reference is not necessary in water because the relation between optical reflectivity and pressure is strictly defined by known material constants.

\section{ACKNOWLEDGEMENTS}

We are grateful to the "Patentstelle für die Deutsche Forschung der Fraunhofer-Gesellschaft" for financial support.

\section{REFERENCES}

[1] Brümmer F., Staudenraus J., Hülser D.F., Eisenmenger W., Physikalische Grundlagen sowie medizinische und biologische Wirkungen unterschiedlich generierter Stoßwellen im Hinblick auf die klinische Anwendung, BMFT final report (1994)

[2] Brümmer F., Wurster C., to be published

[3] Born and Wolf, Principles of Optics, $5^{\text {th }}$ edition, Pergamon Press (1975)

[4] Rosen, J.S., The Refractive Index of Alcohol, water, and their Mixtures at High Pressures, J. Opt. Soc. Am. 37, 932 (1947)

[5] Beyer, R.T.,Nonlinear Acoustics, U.S. Naval Sea Sys. Comm., Washington D.C.(1974)

[6] Müller M., Stoßwellenfokussierung in Was. ser, thesis, RWTH Aachen (1974)

[7] Eisenmenger W., Oszillographische Wiedergabe von Stoßfronten in Flüssigkeiten bei Druckanstiegszeiten von $10^{-9} s$, Proceedings $5^{e}$ Congres International D'Acoustique, part I, K25 (1965)

[8] Staudenraus J., Eisenmenger W., Fiber optic probe hydrophone for ultrasonic and shock wave measurements in water, Ultrasonics, 31, 267 (1993) 